AFMRD from the asscatition of Family Medicine Residency Directors Residency Directors

\section{GETTING THE MOST FROM THE ACGME ANNUAL RESIDENT AND FACULTY SURVEY}

The Accreditation Council for Graduate Medical Education (ACGME) requires all residency programs, within every specialty, to participate in an annual survey of residents and faculty.

The ACGME uses the results of these surveys to monitor graduate medical education quality and provide an early warning for non-compliance to ACGME standards. Residency programs are expected to use the survey results in their Annual Program Evaluation process to assist in each program's own continuous improvement. Aggregate data, featuring a comparison of national and specialty averages, is shared with each program. Since both surveys are now administered early in the calendar year with results sent to programs by May, data are returned in a time frame that fits into the annual program review cycle for most programs.

Specific survey questions are available only to those participating in the survey $y_{i}$ historically the content has focused on the common program requirements of clinical experience and education (formerly duty hours), faculty, evaluation, education content, resources, patient safety, and teamwork. The family medicine resident/fellow survey also contains a secondary set of questions specific to the specialty area. These questions generally focus on continuity, care across settings, and family-centered care.

Residency programs can reap the greatest benefits from these ACGME surveys by taking specific action before the surveys are administered and after the survey results are received.

Survey results are most valuable if the participants, by fully understanding the questions and definitions, respond with knowledge of the context of the questions. To help residents, fellows, and faculty better understand the ACGME surveys, many program directors meet with participants before the surveys to ensure they understand the questions and the nature of the surveys. The purpose is not to sway their answers, only to clarify. This also provides an opportunity to remind residents and faculty of their options to report concerns about the program within their own residency educational system.
The ACGME provides a variety of explanatory documents for both surveys on their website (http:// www.acgme.org/Data-Collection-Systems/ResidentFellow-and-Faculty-Surveys). These documents include survey question content areas, instructions for accessing the survey, and other documents.

Reviewing with participants ACGME's key terms alone is time well spent, as program directors often discover that the low ratings on a particular question may have stemmed from a misunderstanding of the terms, not from a true problem in the program. For example, the residents should understand the difference between "in-house call" and "night float" as described in the ACGME Common Program Requirements. Likewise, residents may find an understanding of the requirement differences between in-house call and at-home call useful as they answer the questions. Regarding the balance between service and education, the ACGME has used the explanation that "one measure of a balance of service and education is that programs avoid routine reliance on residents to carry out activities that do not require a physician." Additionally, program directors may want to point out that the 5-point scale may flip between positive and negative responses being at the top or the bottom.

Once the residency program receives the survey results, program directors have opportunities to use the data within their program. If a program's aggregate data are below the specialty norms, program directors are expected to take action-to understand the factors involved in the survey rating and to lead efforts to make improvements in the program. Program directors may find it helpful to create their own anonymous program survey with a more open-ended format to allow for qualitative comments to help explain the quantitative results of the ACGME survey. Additional ideas for addressing specific issues include conducting a focused survey regarding the identified issue(s), having a chief resident collect comments in a resident-only forum, or using a facilitated small group to help identify details about the issues. In some programs, these will become tasks of the Program Evaluation Committee.

If a program director has prepared participants to understand the ACGME surveys - through discussions and by distributing and reviewing ACGME survey prep documents-the resulting data will offer helpful insight and assist in the Annual Program Evaluation process. Ultimately, the ACGME's annual surveying of residents, fellows, and faculty can serve as powerful tools for residency program improvement, particularly when the meaning of the results is clear.

Karen B. Mitchell, MD, and Thomas Miller, MD 